\title{
Neurophysiologie de l'occlusion : rôle des mécanorécepteurs parodontaux
}

\section{Neurophysiology of occlusion: role of periodontal mechanoreceptors}

\section{Résumé}

\section{MOTS-CLEFS :}

- Mécanorécepteurs parodontaux, mastication, occlusion

\section{KEYWORDS:}

- Periodontal mechanoreceptors, mastication, occlusion

AOS $n^{\circ} 290-2018$
Cet article s'intéresse ò la neurophysiologie de la manducation. Il fait un point sur les connaissances physiologiques actuelles autour des mécanorécepteurs de la bouche et du visage dont les spécifiques mécanorécepteurs parodontaux afin de comprendre leurs différentes fonctions sensori-motrices.
Abstract

This article focuses on the neurophysiology of manducation. He made a point on the current physiological knowledge around the mechanoreceptors of the mouth and the face including the specific periodontal mechanoreceptors in order to understand their different functions.

Gauthier CAZALS, Docteur en chirurgie dentaire-gauthiercazals@hotmail.fr

\section{INTRODUCTION}

L'occlusion dentaire est souvent considérée comme un domaine complexe par les chirurgiens-dentistes. Quelle est la fonction de l'occlusion dentaire? Que se passe-t-il du point de vue neurophysiologique lorsque les dents entrent en contact ? Comment est régulée la manducation (mastication et déglutition) ? Ce premier article en rappelant les fondamentaux physiologiques, vise à apporter les informations actuelles sur les mécanismes périphériques impliqués dans le contrôle de l'occlusion (Fig. 1). Les articles suivants (articles 2 et 3) traitent des contrôles moteurs segmentaires et supra-segmentaires de l'occlusion tant en denture naturelle qu'en présence d'une réhabilitation implanto-portée. Enfin, le dernier article (article 4) consiste en une actualisation des connaissances sur les interférences occlusales.

Les mouvements fonctionnels de l'appareil manducateur et les forces qu'il reçoit dépendent des signaux provenant de divers organes sensoriels répartis dans l'ensemble des structures oro-faciales (dents, mâchoires, articulations temporo-mandibulaires [ATM], muscles, muqueuse orale et téguments de la 


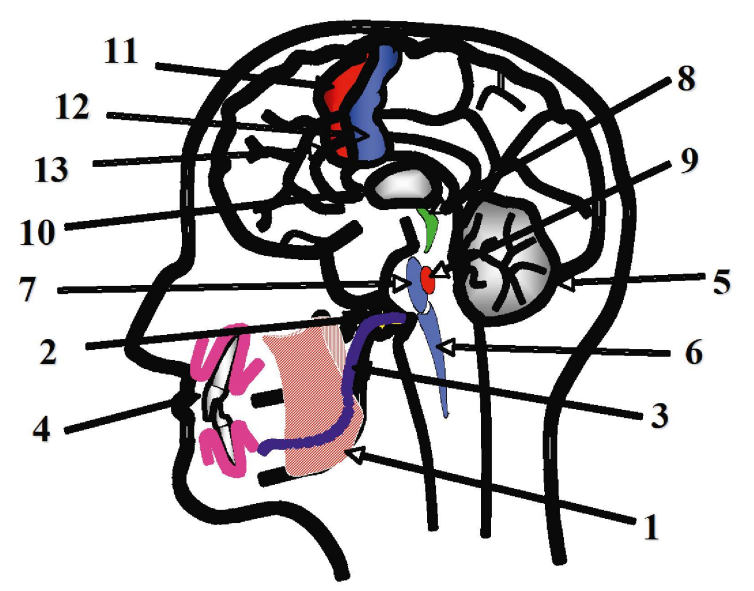

face). Les mécanorécepteurs parodontaux (MRP) et leur innervation sensorielle jouent ici un rôle particulier. Leur localisation dans le ligament parodontal leur permet de détecter les forces qui sollicitent les dents et les mâchoires, par le jeu de la mécano-transduction (transformation du signal physique initial en messages nerveux). Ces récepteurs ont également un rôle dans le contrôle moteur de la mastication et de la déglutition. À ce jour pourtant, nos connaissances dans ce domaine sont encore limitées. En effet, la mastication est un processus dynamique, or les études sur les MRP sont généralement effectuées dans des conditions statiques. De plus, la grande majorité des études est réalisée chez l'animal dont les caractéristiques anatomiques et fonctionnelles peuvent être très différentes de celles de l'Homme.

Il existe différents types de mécanorécepteurs dans l'organisme. À la différence des terminaisons libres (non encapsulées), ce sont des récepteurs dits spécialisés. Dans les téguments cutanéo-muqueux, ils sont représentés par les corpuscules de Pacini, de Ruffini et de Meissner et les disques de Merkel. Dans les muscles ce sont les fuseaux neuromusculaires (FNM) et les organes tendineux de Golgi (OTG). Les articulations telles que les ATM contiennent aussi des récepteurs spécialisés. À côté de ces mécanorécepteurs, ces différents tissus contiennent aussi des terminaisons libres. Les MRP constituent une classe particulière des mécanorécepteurs à l'image de ceux des téguments qui sont biens connus.

\section{LA MÉCANO-TRANSDUCTION}

Les interactions mécaniques des cellules avec leur environnement sont très utiles dans le développement et le fonctionnement de l'organisme. La mécano-transduction se définit comme le phénomène permettant à une cellule de recevoir une information mécanique sur sa membrane et de fournir une $\varangle$ Fig. 1 : Ce schéma situe sur une coupe anatomique sagittale les principales structures participant lors de la mastication.

1. Muscles élévateurs (dont les masséters).

2. Ganglion trigéminal.

3. Nerf alvéolaire inférieur.

4. Dents/Implants et parodonte.

5. Cervelet.

6. Noyau spinal du V (Tronc cérébral).

7. Noyau principal du $\mathrm{V}$ (Tronc cérébral).

8. Noyau mésencéphalique du $\mathrm{V}$ (Tronc cérébral).

6.7.8. Complexe Sensitif du Trijumeau (CST).

9. Noyau moteur du V.

10. Thalamus.

11. Zone motrice M1 (cortex moteur).

12. Zone somesthésique S1 (cortex somesthésique).

13. Aire corticale de la mastication.

réponse adaptée. Elle est un processus dynamique grâce à la capacité des cellules à modifier en temps réel leurs points d'ancrage au substrat. Ce ré-arrangement des protéines impliquées dans l'adhérence permet à la cellule de modifier son comportement. Ainsi, à la suite de l'application d'une force sur un organe, se déclenche une cascade d'évènements moléculaires, à ce jour non élucidés, captés par des récepteurs.

\section{LES MÉCANORÉCEPTEURS DE LA BOUCHE ET DU VISAGE}

Un récepteur est une terminaison sensorielle assurant la transduction, c'est-à-dire la transformation d'une énergie (physique, chimique ou thermique) en messages nerveux. La transduction représente la première étape de la détection d'un signal assuré par le récepteur. Pour un stimulus mécanique, la séquence des événements est la suivante: déformation membranaire, ouverture des canaux ioniques, entrée de $\mathrm{Na}^{+}$et dépolarisation membranaire locale. Puis intervient la transmission des messages qui consiste en la propagation des potentiels d'action le long de la fibre sensorielle dite aussi afférence primaire qui se termine dans le premier relais central. En ce qui concerne la face et la cavité orale, ces messages sont transmis au complexe sensitif du trijumeau (premier relais central). De là, ces messages peuvent avoir deux rôles possibles. Ils peuvent être transmis plus rostralement dans le système nerveux central (SNC) jusqu'au cortex sensoriel primaire (aire S1) de la face, ils ont ainsi un rôle extéroceptif (perceptif). Sinon ces messages par leur rôle proprioceptif participent aux régulations sensitivo-motrices en modulant l'activité des motoneurones qui commandent les fibres musculaires. Ces régulations motrices sont de deux types : soit courtes et segmentaires, soit longues et suprasegmentaires (voir article 2). 
Les mécanorécepteurs sont reliés à des fibres afférentes myélinisées de gros diamètre $(A \beta)$, de conduction rapide. Pour la peau de la face et la muqueuse buccale, ces fibres sont regroupées en faisceaux constitutifs du nerf trijumeau.

Les mécanorécepteurs cutanés sont classés en fonction de la taille de leur champ récepteur et de leur vitesse d'adaptation. Le champ récepteur peut être petit (avec des limites nettes) ou grand (avec des limites imprécises). La vitesse d'adaptation s'observe lorsqu'un stimulus est maintenu constant pendant un certain temps. On distingue ainsi 2 vitesses différentes d'adaptation :

I nulle ou lente qui renseigne sur la valeur absolue de l'intensité du stimulus et sur sa durée. Ce sont des récepteurs toniques ou statiques ;

$\checkmark$ rapide qui traduisent les variations du stimulus en fonction du temps. Ce sont des récepteurs phasiques ou dynamiques.

En général, les mécanorécepteurs qui innervent la peau de la face ainsi que les muqueuses labiale et buccale ont des propriétés similaires aux mécanorécepteurs qui innervent la main de l'homme, c'est à dire les quatre types de mécanorécepteurs décrits au niveau de la peau glabre (Fig. 2) et des récepteurs des follicules pileux. Les mécanorécepteurs à adaptation lente avec une forte sensibilité dynamique (c'est à dire qu'ils répondent vigoureusement à l'apparition du stimulus mécanique) ont une décharge irrégulière durant une déformation tissulaire continue et ressemblent aux récepteurs SA I de la peau glabre et poilue de la main de l'Homme. Un autre groupe de mécanorécepteurs à adaptation lente, caractérisés par un taux de décharge régulier et une activité spontanée, est similaire aux unités SA II. Une caractéristique importante des unités SA II est leur sensibilité exquise à l'étirement latéral de la peau, même quand la stimulation est appliquée loin de la zone de sensibilité maximale du champ récepteur. Les mécanorécepteurs à adaptation rapide de la région oro-faciale ressemblent aux unités FA I de la peau glabre de la main. Pour cette région oro-faciale, il est important de remarquer qu'aucun mécanorécepteur rencontré dans les expériences chez l'homme a présenté des réponses similaires à celles des afférences des corpuscules de Pacini (FA II), à l'exception de la face ventrale de la langue; ceci concorde avec les résultats psycho-physiologiques chez l'Homme, et les résultats neurophysiologiques et morphologiques chez d'autres espèces. C'est-à-dire que la région oro-faciale, à la différence de la main, est particulièrement insensible aux vibrations de haute fréquence et mécaniques transitoires, les stimuli pour lesquels les mécanorécepteurs FA II sont les plus sensibles (11).

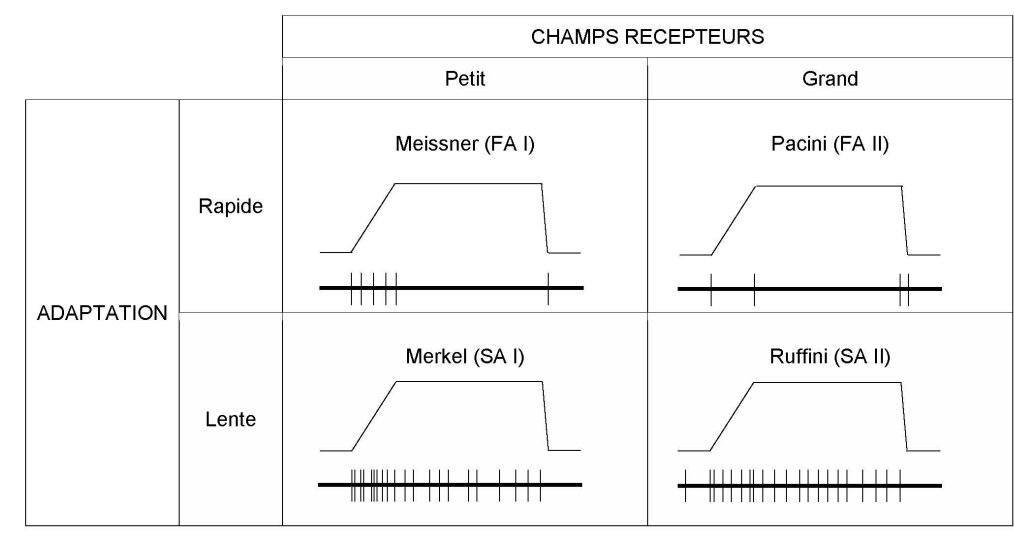

$\triangle$ Fig. 2 : Classification des mécanorécepteurs cutanés basée sur l'adaptation de leurs réponses à une augmentation de la force qui leur est appliquée et sur la taille des champs récepteurs. Pour chaque récepteur, le tracé supérieur montre l'augmentation de force appliquée au récepteur alors que le tracé inférieur montre un tracé typique de réponse du mécanorécepteur à cette force. Tous les récepteurs répondent à l'augmentation de la force mais seuls ceux à adaptation lente continuent de répondre alors que la force est maintenue (réponse statique). (Tableau modifié issu de Trulsson M and Essick GK in Clinical Oral Physiology, 2004. Quintessence).

La plupart des mécanorécepteurs de la peau de la face et du vermillon des lèvres sont à adaptation lente avec des champs récepteurs bien définis et petits. Les mécanorécepteurs à adaptation lente semblent aussi prédominer dans la muqueuse buccale. Par contre, la plupart des mécanorécepteurs se terminant superficiellement dans la langue s'adaptent rapidement à une déformation constante et ont des champs récepteurs extrêmement petits et bien définis. Selon Trulsson et Essick, l'innervation sensitive de la face et des joues ressemble à celle de la peau poilue des mains et des bras alors que l'innervation de la langue ressemble à celle de l'extrémité des doigts (11). Ces différences de l'innervation sensitive sont bien adaptées aux spécialisations fonctionnelles de ces différentes régions. L'extrémité de la langue et celle des doigts servent à manipuler et explorer les objets dans la bouche et avec la main respectivement. Par contre, les mécanorécepteurs innervant la peau de la face et la face dorsale de la main servent à signaler les mouvements de la face et des doigts. Ces mouvements fonctionnels de la face et des doigts produisent des patrons de décharges variant en fonction du temps lors de l'étirement de la peau, ce qui stimule ces récepteurs.

\section{Clinique}

En pratique clinique, on est témoin de l'importance des mécanorécepteurs dans le contrôle moteur de la face et de la bouche. L'anesthésie locale (dentaire) des nerfs sensitifs des mâchoires, des lèvres et de la langue entraine non seulement une perturbation des sensations, dont celles issues des nocicepteurs de la pulpe dentaire, mais aussi une perturbation des activités motrices, en particulier de celles qui nécessitent un contrôle précis de la musculature oro-faciale. Par exemple, la salive coule de la bouche (bavement) du fait de l'incompétence labiale due au manque de contrôle sensitif. 
La forte densité des mécanorécepteurs ayant des champs récepteurs petits et bien définis au niveau de la pointe de la langue et des lèvres est le reflet de la forte résolution spatiale de l'acuité de ces régions.

\section{LES MÉCANORÉCEPTEURS PARODONTAUX}

Le ligament parodontal ancre les racines des dents à l'os alvéolaire. Bien que plutôt envisagé comme un mécanisme de soutien des dents, le ligament parodontal est aussi un organe somesthésique important richement innervé par des mécanorécepteurs de bas seuil. On pense qu'il y a environ 300 afférences mécanoréceptrices innervant le desmodonte de chaque dent chez l'homme (12). Les fibres nerveuses sensitives passent au travers du foramen apical et des foramina latéraux de l'alvéole dentaire et se terminent dans le ligament parodontal. Les terminaisons nerveuses retrouvées sont pour la grande majorité du type de Ruffini, en étroite relation avec les fibres de collagène (Fig. 3). Elles peuvent varier en complexité allant de simples petites terminaisons jusqu'à des terminaisons très ramifiées laissant supposer une diversité de fonctions. Comme pour les récepteurs cutanés, ces récepteurs sont reliés à des fibres afférentes myélinisées de gros diamètre $(A \beta)$, de conduction rapide.

Des études chez le rat ont démontré que la maturation morpho-physiologique des terminaisons parodontales de Ruffini est étroitement liée à l'éruption dentaire (1). L'arborisation finale de ces terminaisons se termine peu après le début de la fonction molaire (15).

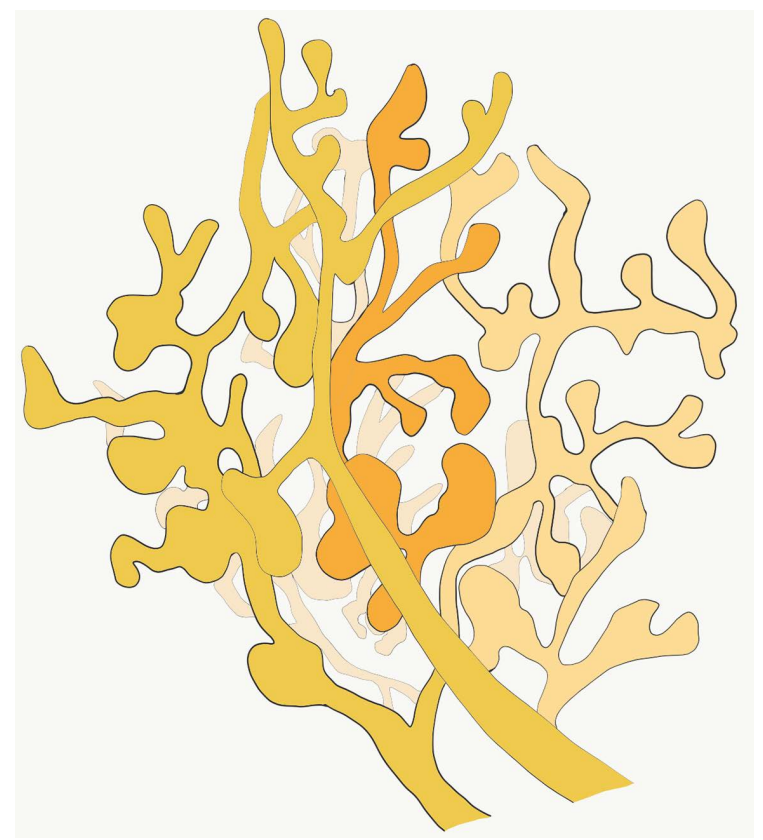

$\triangle$ Fig. 3 : Schéma descriptif d'une terminaison parodontale de type Ruffini. (Dessin issu de Boucher Y in Parodontologie \& Dentisterie implantaire, 2015. Lavoisier).
Chez l'homme, il y a essentiellement deux types d'études sur les MRP, histologiques et électro-physiologiques, avec des conclusions parfois contradictoires. Les études portant sur l'anatomie macroscopique humaine sont rares et limitées à cause de la difficulté à isoler le ligament parodontal humain dans son intégrité. Huang et al ont observé des ligaments parodontaux de canines mandibulaires à partir de cadavres humains. Ces auteurs ont trouvé une innervation dense de fibres nerveuses myélinisées à proximité des fibres de collagène et de l'os alvéolaire. La densité de l'innervation semble augmenter en se rapprochant de l'apex et être plus importante dans les sites apical, mésial et lingual (5). Il y a moins de MRP au niveau des dents postérieures par rapport aux dents antérieures, ce qui atteste de l'importance de l'innervation mécano-réceptrice bien développée du segment antérieur de la denture. Les dents antérieures sont impliquées dans les stades initiaux de la prise de nourriture dans la bouche, et servent à guider la mâchoire vers la position d'intercuspidie durant la dernière phase du cycle de mastication.

Pour Trulsson, les MRP humains apparaissent être presque entièrement de type à adaptation lente. Ils sont localisés généralement autour de la circonférence de la dent et plutôt concentrés dans les zones soumises aux plus grandes forces, particulièrement dans une direction disto-linguale (11). Les récepteurs desmodontaux de Ruffini sont des arborisations non encapsulées, reliées aux fibres de collagène. Typiquement, ces récepteurs ont une taille de 50 micromètres (2). D’autres études histologiques plus récentes sur les fibroblastes du ligament parodontal à partir de prémolaires extraites pour des raisons orthodontiques montrent que la transduction des stimuli mécaniques (stimulation compressive) dans le ligament parodontal fait intervenir la voie des kinases d'adhésion focale (focal adhesion kinase (FAK) pathway) ainsi que le complexe intégrine-FAK qui joue le rôle de mécanorécepteur dans les cellules desmodontales (8).

La plupart des expérimentations sur les humains sont électro-physiologiques. Malheureusement elles sont généralement limitées et souvent déductives, les limitations chez l'homme étant bien plus importantes que pour les animaux. Des études chez l'homme et chez l'animal, enregistrant l'activité électro-physiologique des MRP, ont démontré la présence de deux types de récepteurs basiques, qui répondent tous deux aux mouvements des dents suite à l'application d'une force externe. Un type est appelé unité d'adaptation rapide: il génère des décharges transitoires à un stimulus soutenu, et le nombre d'impulsions produites dépend du taux d'application du stimulus. À des taux d'application plus rapides, plus d'impulsions nerveuses sont 
produites. Ainsi, ils sont sensibles à la fois à la vitesse et à l'ampleur de la charge appliquée, mais ne réagissent que lorsque la charge est appliquée (4). L'autre type est l'unité à adaptation lente qui, au contraire, continue à générer des impulsions nerveuses pendant de longues périodes, tout au long du déplacement de la dent et même si la charge n'est plus appliquée sur la dent. Plus de 40 à $50 \%$ de ces récepteurs sont spontanément actifs produisant des potentiels d'action en l'absence de force appliquée. Dans ces types de récepteurs, la fréquence de l'impulsion au cours de l'initiation de la réponse s'accroît avec une augmentation de l'application du stimulus; les fréquences initiales et finales dépendent également de l'ampleur de la charge appliquée $(4,11)$.

En résumé, ces études avec des conclusions parfois contradictoires nous montrent que les MRP sont des récepteurs spécifiques, avec probablement de nombreuses variations, très proches des mécano-récepteurs de type Ruffini retrouvés au niveau de la main.

\section{STIMULATION DES MRP}

Lors de la mise en charge d'une dent, elle se déplace légèrement dans son alvéole. Cliniquement, on l'observe parfois par le fremitus, le mouvement léger qui peut être ressenti par les doigts quand une dent entre en contact avec les dents antagonistes. Ce mouvement induit des tensions et des contraintes dans le ligament parodontal.

Les mécanorécepteurs sont sensibles à la direction de la force. Par conséquent, chez l'Homme, quand une force est appliquée sur la dent dans une direction, les mécanorécepteurs parodontaux répondent avec quatre ou six afférences différentes, mais l'activation maximale se produit dans le sens du stimulus. Ainsi, l'existence de plusieurs champs réceptifs dentaires ne réduit pas la capacité du système nerveux central à localiser avec précision la charge appliquée à une dent.

Le seuil moyen de perception d'une force capable d'activer un récepteur chez l'Homme est inférieur à $1 \mathrm{~N}$ (13). Par exemple, la quantité de force nécessaire pour casser une cacahuète diffère en fonction du type de dent impliquée. Elle augmente distalement le long de l'arcade dentaire avec une moyenne de $0,60 \mathrm{~N}$ pour les incisives, $0,77 \mathrm{~N}$ pour la canine, $1,15 \mathrm{~N}$ pour les prémolaires et $1,74 \mathrm{~N}$ pour la première molaire. Trulsson et al ont aussi montré que durant l'anesthésie parodontale, l'amplitude et la variabilité de ces forces sont au moins multipliées par 2 pour tous les types de dents. Cela corrobore le fait que les MRP jouent un rôle important pour affiner l'activité musculaire mais ne sont pas les seuls car d'autres récepteurs (FNM, OTG et récepteurs articulaires des ATM essentiellement) assurent la régulation sensori-motrice quand ils ne répondent plus.

Plusieurs études animales soulignent la relation étroite de l'occlusion avec les MRP. Sodeyama et al (9) ont démontré chez des rats, avec des forces occlusales différentes, qu'un traumatisme occlusal induit des changements spécifiques dans la distribution et la forme des terminaisons nerveuses au sein du ligament parodontal; d'autres études sur des rats ont montré qu'une force occlusale incorrecte modifiait de façon réversible les terminaisons axonales et les propriétés fonctionnelles des terminaisons parodontales de Ruffini (8).

\section{VOIES NEURONALES SENSORIELLES DEPUIS LES MRP VERS LE SNC}

Afin de mieux connaître les mécanismes qui contrôlent les forces masticatoires, il est important de comprendre les voies de signalisation provenant des MRP. La localisation des corps cellulaires des neurones primaires qui transmettent les informations des MRP vers le système nerveux central (SNC) est en ce sens très intéressante. Les corps cellulaires des neurones afférents primaires qui répondent aux forces appliquées aux dents ont été détectés dans deux sites anatomiques distincts : le ganglion trigéminal (GT) et le noyau mésencéphalique trigéminal (NM).

Les fibres afférentes mécanosensitives du GT se connectent avec des neurones secondaires dans les noyaux principal et spinal du Complexe Sensitif du Trijumeau (CST). À partir de là, les informations peuvent être relayées au niveau du tronc cérébral pour influencer de manière réflexe les motoneurones du trijumeau, ou plus rostralement au noyau ventro-postéro médian du thalamus, puis vers l'aire corticale de la mastication. La voie neuronale des afférences à travers le GT conduit à des sensations conscientes via l'intégration du thalamus et du cortex alors que la voie neuronale des afférences à travers le NM conduit à des réflexes inconscients via le cervelet.

Les neurones afférents primaires du GT sont retrouvés en plus grande quantité dans les études animales par rapport à ceux issus du NM. Pour Trulsson et Essick, les afférences du TG sont réparties sur tout le long du ligament depuis la gencive marginale vers l'apex de la racine alors que celles du NM sont concentrées près de l'apex de la dent (11). 


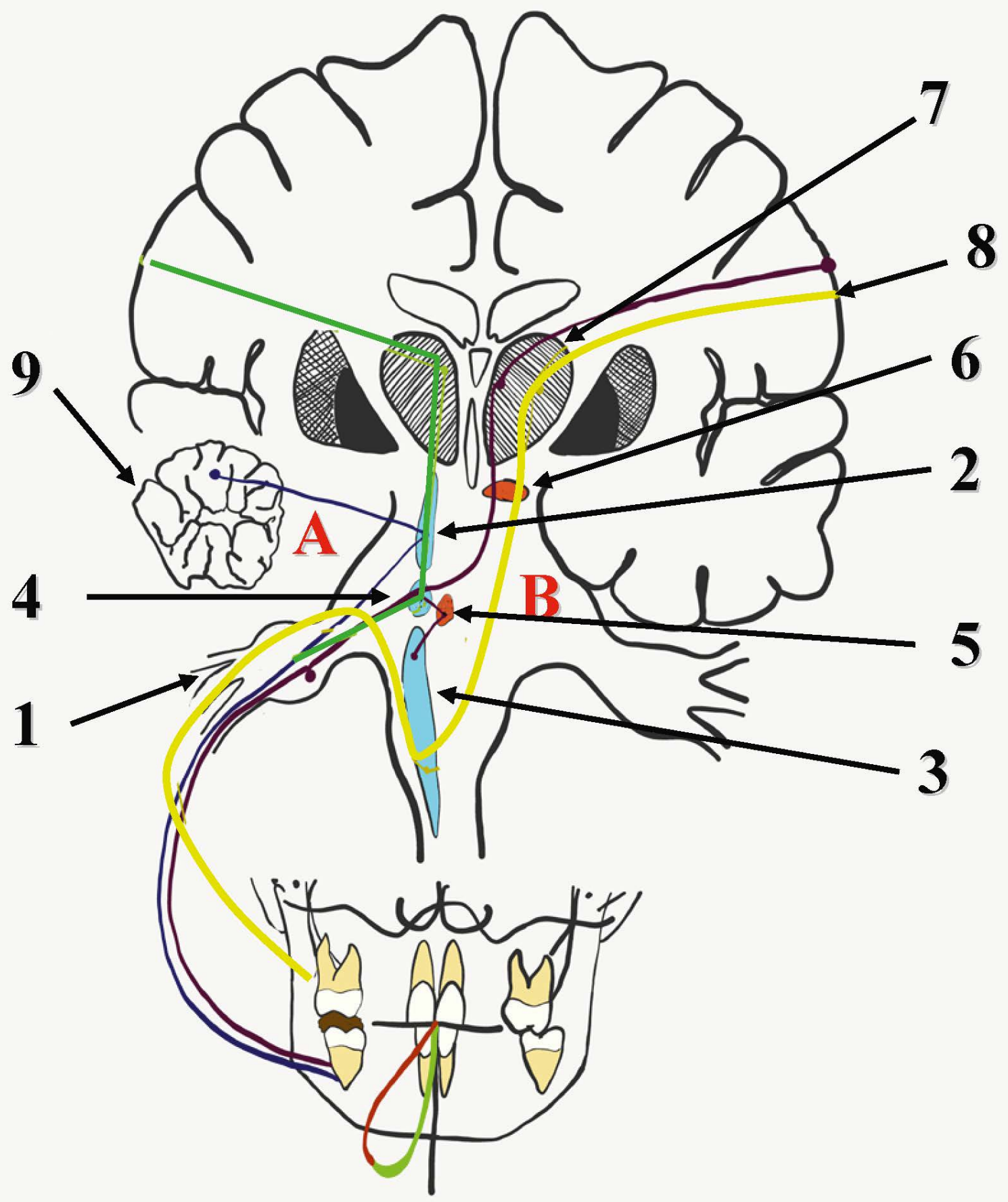

$\Delta$ Fig. 4 : Représentation schématique des voies sensorielles provenant des mécanorécepteurs parodontaux vers le système nerveux central. A : Trajet du neurone afférent primaire localisé dans le noyau mésencéphalique qui conduit aux réflexes inconscients via le cervelet. Ce type de neurone est beaucoup retrouvé au niveau de la canine du chat mais significativement moins dans les autres espèces.

$\mathrm{B}$ : Trajet des neurones afférents primaires localisés dans le ganglion trigéminal (la majorité) qui conduisent à des sensations conscientes, via le thalamus, vers l'aire corticale de la mastication. Les influx sensoriels provenant des mécanorécepteurs sont très puissants dans l'adaptation constante du générateur de rythme (CPG).

1 : Ganglion Trigéminal.

2 : Noyau mésencéphalique du $\mathrm{V}(\mathrm{CST})$.

3 : Noyau spinal du V (CST)

4 : Noyau principal du $\mathrm{V}$ (CST).

5 : Noyau moteur du V.

6 : Générateur Central de Rythme (CPG)

7 : Thalamus.

8: Aire corticale de la mastication.

9: Cervelet.

(Schéma issu de Piancino et al. AOB 2017). 


\section{RÔLES DES MRP}

Il est bien établi que l'activité motrice de la mastication est générée par des réseaux de neurones dans le tronc cérébral en l'absence d'information somesthésique de la bouche et de la face (voir article 2) et il est maintenant prouvé que les signaux provenant des afférences mécanoréceptrices régulent l'activité musculaire manducatrice (3).

Premièrement, les MRP apportent des influx aux voies réflexes inhibitrices et excitatrices bien caractérisées. La voie réflexe inhibitrice correspond à l'inhibition rapide des motoneurones lorsqu'une force vive est reçue par les MRP (aliment dur). Ainsi, l'inhibition des motoneurones des muscles élévateurs fait cesser leur contraction ce qui évite un nouvel impact sur l'aiment dur. Cette réponse réflexe protectrice prend environ 0,01 s pour agir. Inversement la voie réflexe excitatrice correspond à l'activation des motoneurones des muscles élévateurs, par une population différente de MRP. La fonction de ce réflexe excitateur n'est pas entièrement connue. Cependant, il semble probable que son rôle est d'aider les muscles masticateurs à garder l'aliment entre les dents. Quand les dents occluent sur un aliment mou durant la mastication, les MRP sont activés. Les signaux qu'ils envoient aux motoneurones trigéminaux de façon réflexe activent les muscles élévateurs qui se contractent un peu plus fort pour maintenir le bol alimentaire entre les dents qui sont alors prêtes à le broyer (6).

D'autres preuves, issues d'études comportementales chez des patients avec et sans MRP, démontrent que les MRP sont normalement des récepteurs qui dictent les intensités de force de fermeture quand les substances sont manipulées et maintenues légèrement entre les dents. Etant donné la sensibilité élevée pour des forces faibles de la plupart des afférences parodontales, celles-ci sont particulièrement adaptées à signaler des informations sur l'aliment alors qu'il est positionné pour être mâché et durant la phase de contact précoce de chaque cycle de mastication. Au cours de cette période, quand les forces de fermeture sont faibles, les informations relatives à la distribution des particules alimentaires et leurs propriétés intrinsèques sont collectées, traitées par le SNC et utilisées pour réguler l'activité subséquente des muscles de la mâchoire. Ceci a été montré dans des expériences de laboratoire chez l'Homme, où l'on a trouvé que des mécanismes anticipatoires ou prédictifs ajustent les forces de la mâchoire à l'impédance mécanique d'un aliment test au cours des mouvements de mastication. C'est-àdire qu'une partie des forces durant la fermeture était déterminée de façon prédictive depuis les expériences somesthésiques antérieures concernant l'aliment. Les expériences somesthésiques, qui sont sans doute en partie d'origine parodontale, ont été acquises au cours du cycle de mastication précédent et aident ensuite à déterminer l'activité musculaire supplémentaire nécessaire durant la phase de puissance de la mastication pour surmonter la résistance de l'aliment. On considère ce contrôle prédictif comme un contrôle en boucle ouverte, (open-loop control ou feedforward), puisqu'il ne dépend pas d'un contrôle continu de la capacité des dents à broyer le bol alimentaire. Le contrôle prédictif diminue la nécessité du rétro-contrôle dit aussi contrôle en boucle fermée, (closed-loop control ou feedback) qui utilise les signaux issus des FNM des muscles masticateurs (voir article 2). En général, les mécanismes de contrôle moteur en boucle fermée sont plus précis que les contrôles en boucle ouverte, mais ils nécessitent plus de temps pour fonctionner correctement. Durant la mastication lente, il a été estimé que le contrôle prédictif explique environ seulement $15 \%$ de l'ajustement de la force à la résistance du bol alimentaire. Le rétro-contrôle durant la fermeture (c'est-à-dire le contrôle réactif) explique la plupart, soit $85 \%$ de l'adaptation de la force quand le temps n'est pas un facteur limitant. Cependant, durant la mastication rapide (environ 120 cycles/minute) le contrôle prédictif contribue à plus de la moitié (60 \%) de l'ajustement de la force (11).

D'autre part, selon Piancino, l'importante influence des MRP sur le Générateur Central de Rythme ou CPG (Central Pattern Generator) a été clairement démontrée et pourrait expliquer les cycles de mastication inverses (reverse sequencing chewing cycles) qui sont fortement retrouvés chez des patients avec un articulé inversé postérieur lorsqu'ils mastiquent de ce côté. Un articulé inversé postérieur est caractérisé par des relations inversées entre les cuspides des molaires. La fonction masticatoire qui en résulte, appelée modèle de mastication inverse (reverse chewing pattern), entraine une direction inverse de fermeture, assurant ainsi le chevauchement des surfaces occlusales dentaires opposées (8). C'est-à-dire que les sujets ayant un articulé inversé postérieur unilatéral ont un fonctionnement musculaire différent lors de la mastication en fonction de la position du bolus alimentaire. Lorsque ce dernier se trouve du côté de l'articulé inversé, la mandibule dévie d'abord médialement lors de la fermeture puis latéralement assurant le chevauchement des surfaces occlusales dentaires opposées. Inversement, lorsque le bolus est du côté controlatéral, la mandibule dévie d'abord latéralement puis médialement lors de la fermeture. Dans cette étude, ils rapportent une diminution significative de l'activité électromyographique du masséter du côté de l'articulé inversé en comparaison avec le masséter controlatéral (8). Cela est cohérent 
avec la localisation et les caractéristiques des MRP dont les informations contribuent à établir le schéma le plus pratique et conservateur d'un point de vue énergétique, particulièrement lors de la puissante phase de fermeture de la mastication.

En plus de leur rôle dans le contrôle moteur de la mâchoire, les MRP sont impliqués dans la détection des objets entre les dents. Des études sur la capacité de détection d'objets de différentes épaisseurs montrent une formidable compétence des MRP à détecter de petites variations. Des sujets avec des dents naturelles maxillaires et mandibulaires sont capables de détecter des feuilles de 20 micromètres d'épaisseur. Lorsque le patient porte des prothèses complètes rétentives (absence des MRP), le seuil minimal de détection de la feuille est 7 à 8 fois supérieur. Si la rétention est moins bonne, le seuil augmente encore. Il est à noter le cas des dents traitées endodontiquement qui ont un sensibilité proche mais moins bonne que celle des dents naturelles, laissant supposer un impact du traitement sur les MRP et/ou un rôle des mécano-récepteurs pulpaires.

Ainsi, même si les études sont difficiles à réaliser chez l'homme et que souvent dans les publications le terme de MRP regroupe d'autres récepteurs comme ceux de la bouche et du visage ou encore ceux des ATM, il semble acquis que les MRP ont un rôle primordial dans le contrôle moteur de la mâchoire, réflexe, prédictif ou rétroactif, dans la direction des forces exercées par la mâchoire et dans la sensibilité tactile des dents (14).

\section{CONCLUSION}

Aucune autre région du corps ne présente un système somesthésique aussi développé que celui de la face et de la cavité orale. C'est pourquoi la perception orofaciale des stimulations tactiles est la plus fine de tout l'organisme. L'appareil manducateur fait preuve d'une remarquable capacité d'adaptation à un environnement biomécanique en mutation, qu'il s'agisse de changements de structure ou de demandes fonctionnelles (7). Les connaissances actuelles des MRP nous font conclure que ce sont des récepteurs neuronaux très sophistiqués profondément impliqués dans l'activation et la coordination des muscles masticateurs. Attachés aux dents, ils permettent des processus corticaux sensori-moteurs adaptatifs et compensatoires. Leur grande sensibilité aux forces faibles leur permet de coder les changements temporels dans la force de mastication lors de la phase de contact occlusal des cycles masticatoires, ce qui permet d'apprécier la texture des aliments (rôle extérocepteur) et de préparer les cycles suivants (rôle propriocepteur).

Ces fonctions sensori-motrices importantes sont perdues ou altérées lorsque les dents sont extraites. Cette connaissance souligne l'importance de maintenir les dents naturelles avec une fonction parodontale saine autant que possible (10). 


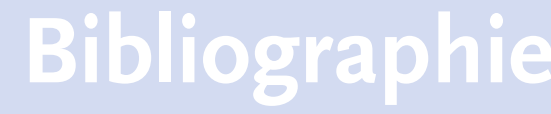

[1] Asahito T, Ohshima H, Hanada K, Wakisaka S, Maeda T. Postnatal expression of calretinin-immunoreactivity in periodontal Ruffini endings in the rat incisor: A comparison with protein gene product 9.5 (PGP 9.5)-immunoreactivity. Arch Histol Cytol 1999; 62(1): 57-69.

[2] Boucher Y, Braud A. Innervation et microcirculation parodontale. in Parodontologie \& Dentisterie implantaire (volume 1). Lavoisier, 2015.

[3] Fougeront N, Garnier B, Fleiter B. Automatismes de l'appareil manducateur et fonctions cervicales connexes ( $2^{\mathrm{e}}$ partie). Med Buccale Chir Buccale 2014; 20:253-261.

[4] Hannam AG. Receptor fields of periodontal mechanosensitive units in the dog. Arch Oral Biology 1970; 15(10):971-78.

[5] Huang Y, Corpas LS, Martens W, Jacobs R, Lambrichts I. Histomorphological study of myelinated nerve fibres in the periodontal ligament of human canine. Acta Odontol Scandin 2011; 69(5):279-286.

[6] Miles TS. Mastication in Clinical Oral Physiology. Quintessence Publishing, 2004.

[7] Peck CC. Biomechanics of occlusion Implications for oral rehabilitation. J Oral Rehabil 2016; 43(3): 205-214.

[8] Piancino MG, Isola G, Cannavale R, Cutroneo G, Vermiglio G, Bracco P, Anastasi GP. From periodontal mechanoreceptors to chewing motor control: a systematic review. Arch Oral Biology 2017; 78:109-121.

[9] Sodeyama T, Maeda T, Takano Y, Hara K. Responses of periodontal nerve terminals to experimentally induced occlusal trauma in rat molars: Animmunohistochemical study using PGP 9.5 antibody. J Periodont Res 1996; 31(4):235-48.

[10] Trulsson M. Force encoding by human periodontal mechanoreceptors during mastication. Arch Oral Biology 2007; 52(4):357-60.
[11] Trulsson M, Essick GK.

Mechanosensation in Clinical Oral Physiology. Quintessence Publishing, 2004.

[12] Trulsson M, Essick GK. Sensations evoked by microstimulation of single mechanoreceptive afferents innervating the human face and mouth. J Neurophysiol 2010; 103(4):1741- 47.

[13] Trulsson M, Van der bilt A, Carlsson GE, Gotfredsen K, Larsson P, Muller F, Sessle BJ, Svensson P. From brain to bridge: masticatory function and dental implants. J Oral Rehabil $2012 ; 39: 858-77$.

[14] Türker KS. Reflex control of human jaw muscles. Crit Rev Oral Biol Med 2002;13(1):85-104.

[15] Umemura T, Yasuda K, Ishihama K, Yamada H, Okayama M, HasumiNakayama Y, Furusawa K. A comparison of the postnatal development of muscle-spindle and periodontal -ligament neurons in the mesencephalic trigeminal nucleus of the rat. Neuroscience Letters 2010;473(2):155-157. 